\title{
Repair or Progression after AKI: A Role for Biomarkers?
}

\author{
Nattachai Srisawat ${ }^{a, b}$ Raghavan Murugan ${ }^{b} \quad$ John A. Kellum ${ }^{b}$ \\ ${ }^{a}$ Excellence Center for Critical Care Nephrology, King Chulalongkorn Memorial Hospital, Thai Red Cross, and \\ Division of Nephrology, Department of Medicine, Faculty of Medicine, Chulalongkorn University, Bangkok, Thailand; \\ ${ }^{b}$ The Center for Critical Care Nephrology, CRISMA (Clinical Research, Investigation, and Systems Modeling of Acute \\ Illness) Center, Department of Critical Care Medicine, University of Pittsburgh School of Medicine, Pittsburgh, Pa., USA
}

\section{Key Words}

Acute kidney injury $\cdot$ Renal recovery · Biomarkers

\begin{abstract}
Recent progress in biomarkers represents a paradigm shift in acute kidney injury (AKI) research. Most studies have evaluated the use of these biomarkers for early diagnosis of AKI. However, the role of novel biomarkers in predicting renal recovery, though less understood, holds great clinical promise. Accurate prediction would help physicians distinguish patients with poor renal prognosis in whom further therapy is unlikely to be useful from those who are likely to have good renal prognosis. Unfortunately, current general clinical severity scores (APACHE, SOFA, etc.) and AKI-specific severity scores are not good predictors of renal recovery. The biology of renal recovery requires the repopulation by surviving renal tubular epithelial cells with the assistance of certain renal epithelial cell and specific growth factors such as neutrophil gelatinase-associated lipocalin (NGAL), hepatocyte growth factor (HGF), epidermal growth factor, and insulinlike growth factor-1 (IGF-1), etc. These markers play a major role in the recovery process. This review will describe the mechanisms of the renal recovery, epidemiology, the role of
\end{abstract}

conventional clinical predictors and finally the role of novel biomarkers (NGAL, HGF, IL-8, IL-18, TNFR-1, IGF-binding protein-7 and tissue inhibitor of metalloproteinase-2) in predicting renal recovery.

(c) 2014 S. Karger AG, Basel

\section{Introduction}

How to facilitate renal recovery is a key question in the management of acute kidney injury (AKI). Two large randomized controlled trials, i.e. the Acute Renal Failure Trial Network (ATN) study [1] and the Randomized Evaluation of Normal versus Augmented Level (RENAL) renal replacement therapy study [2], showed that a significant number of AKI patients failed to return to baseline renal function following an episode of AKI. Importantly, the rate of recovery was 3 -fold different across these two trials, suggesting that the risk of nonrecovery is variable and is perhaps affected by treatment. In this pa-

Changing Paradigms in Acute Kidney Injury: From Mechanisms to Management - Proceedings of the 5th Annual UAB-UCSD O'Brien Center Symposium (San Diego, Calif., USA, March 4, 2014).

\section{KARGER}

E-Mail karger@karger.com www.karger.com/nec
(C) 2014 S. Karger AG, Basel

1660-2110/14/1274-0185\$39.50/0
John A. Kellum, MD, FCCM

Department of Critical Care Medicine

University of Pittsburgh

608, Scaife Hall, 3550 Terrace Street, Pittsburgh, PA 15261 (USA)

E-Mail kellumja@ccm.upmc.edu 
Fig. 1. Process of renal recovery after an acute insult to tubular epithelial cells [37]. $\mathrm{N}$-cadherin = Neural cadherin; E-cadherin = epithelial cadherin; $\mathrm{ZO}-1$ = zona occludens; $\alpha$-SMA $=\alpha$-smooth muscle actin; FSP-1 = fibroblast-specific protein-1.

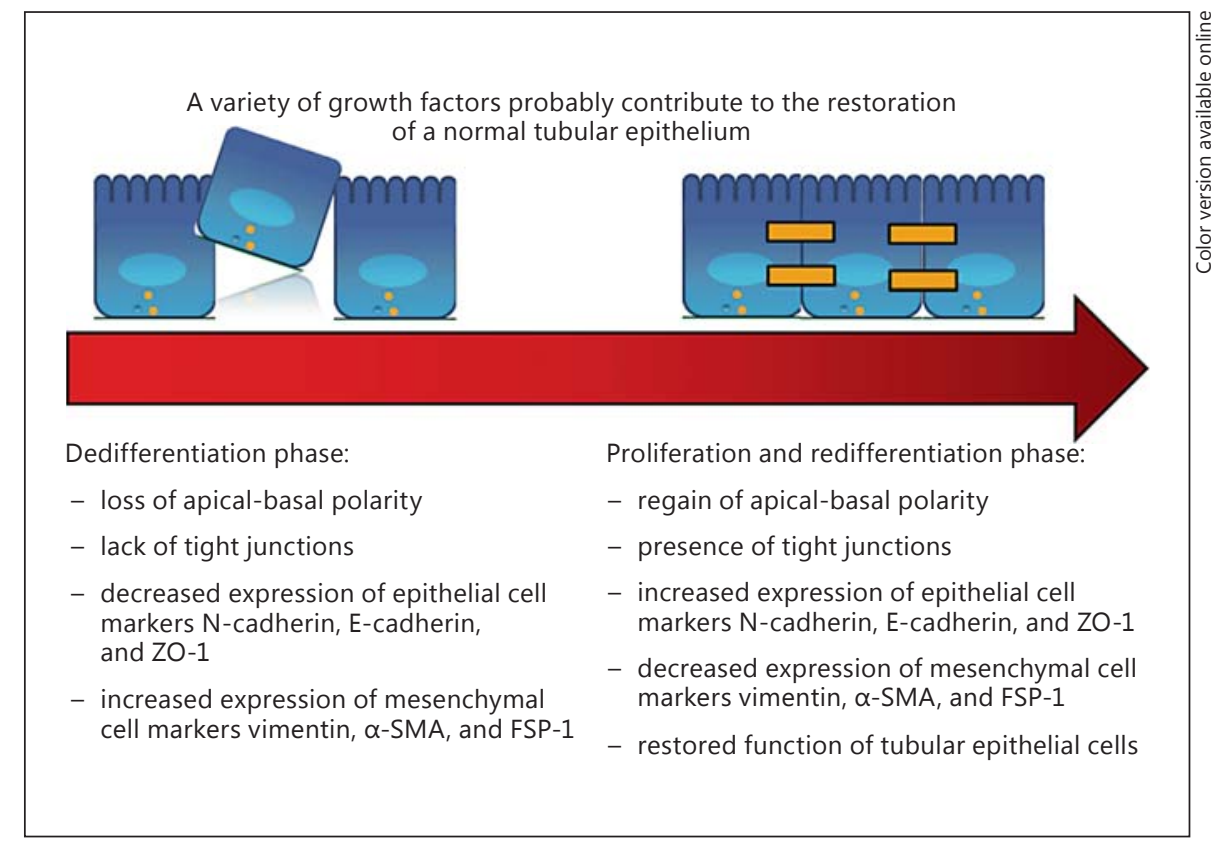

per we will explore the definition, mechanism, epidemiology, and outcome predictors of renal recovery with the goal of improving AKI outcomes.

\section{Mechanisms of Renal Recovery and Repair}

The pathobiology of AKI appears to involve a complex interplay between tubular injury, renal hemodynamics, and inflammation. Surviving renal proximal tubular epithelial cells play a major role in the repair process and appear to follow a program which shares a number of similarities with the series of events observed during kidney development. Renal proximal tubular epithelial cells first appear to undergo dedifferentiation (i.e. loss of apical-basal polarity and a lack of tight junctions accompanied by a decrease in the expression of epithelial cell markers and an increase in the expression of mesenchymal cell or fibroblast markers) and then proliferation. When the cell population has expanded sufficiently to physically replenish the sloughed epithelium, the cells undergo a redifferentiation process characterized by a decrease in mesenchymal cell markers and an increase in epithelial cell markers to finally restore the physiologic function of renal proximal tubular epithelial cells [4].

A number of growth factors such as hepatocyte growth factor (HGF), epidermal growth factor, insulin- like growth factor-1 (IGF-1), bone morphogenic protein-7, and transforming growth factor- $\beta$ help renal repair by interacting with the transmembrane receptors of the tubular epithelial cells. Ultimately, the renal repair process requires redifferentiation of transformed renal epithelial cell function to restore tubular morphology (fig. 1) [5].

Various other mechanisms have been implicated in the control of the repair process [6], and many of these could be exploited as diagnostic or therapeutic targets. Recently, a cell-cell communication mechanism known as Notch, which regulates development, tissue homeostasis, and repair, was identified to play a potential role in repair and regeneration after AKI [7]. Generally absent in the mature kidney, Notch is reactivated after AKI and could be responsible for cellular differentiation, proliferation, and repair [8]. This notion is supported by studies from various laboratories independently observing a linkage between an increased expression of Notch2 and its target Hes 1 and an increased proliferation of renal tubular epithelial cells in the setting of various insults [911].

Another important mechanism is cell-cycle regulation. When epithelial cells, including those lining the proximal tubule, become injured or even stressed, they may deviate from normal cell cycle progression. Cells that become 'arrested' at G2/M may adopt a proinflammatory phenotype that is also profibrotic [12]. There may also be 
a therapeutic value in blocking the initiation of G2/M arrest or using various agents to stimulate cell cycle progression $[13,14]$.

\section{Epidemiology of Renal Recovery}

Studies of AKI outcomes have been heterogeneous in terms of patient populations, etiologies of AKI, outcomes, and the duration of follow-up. We can divide the studies into 2 major groups based on the study populations. First, studies have focused on patients who receive renal replacement therapy (RRT), and they have considered renal recovery as dialysis independence. Almost all studies of this type have been conducted in the ICU setting. AKI outcomes from the major clinical trials show mortality rates ranging from 35 to $60 \%$ [1, 2, 14-18]. Unfortunately, these trials did not necessarily report how many patients died while still receiving RRT. The renal recovery rate among survivors of severe AKI (dialysis independence) varied from 75 to $90 \%$. However, these statistics hide the fact that survival following severe AKI is poor and more patients die following discharge than enter chronic dialysis. Second, studies have focused on AKI more broadly and have performed long-term follow-up (more than 2 years) [19-28]. Most of these studies have included AKI across all severity groups, not only severe AKI. Outcomes have usually included mortality, CKD, and end-stage renal disease rates (per 100 person-years). The major obstacle to drawing conclusions from these studies with regard to the relationship between AKI and CKD or endstage renal disease is the presence of various confounders that can affect the long-term outcomes after AKI.

\section{Clinical Predictors of Recovery}

Clinical severity scores are one set of current tools for predicting renal recovery. Uchino et al. [29] tested 2 general illness severity scores (APACHE II and SOFA) and 4 AKI-specific severity scores in 1,742 patients as part of the BEST Kidney Study. Unfortunately, neither of these scoring systems had a high level of discrimination or calibration to predict outcomes for AKI patients. Thus, though not specifically tested, it seems unlikely that any of the current severity scores can provide a good prediction of renal recovery. We still need a study that is directly designed to identify the factors associated with renal recovery.

Urine output is one of the conventional and oldest known biomarkers. Although oliguria is a common event in the ICU and urine flow is one of the diagnostic and staging criteria for AKI, only a few studies have focused on the role of urine output as a prognostic renal biomarker. A recent study by Macedo et al. [30] found that oliguric patients without a change in serum creatinine have an increased mortality, increased dialysis requirements, and longer ICU and hospital stays than patients without AKI. Subsequent analysis data from the BEST kidney study [31] found that urine output had an area under the receiver operating characteristics curve for successful discontinuation of continuous RRT of 0.85 - although it fell to 0.67 when diuretics were used.

\section{Novel Biomarkers for Recovery}

Based on the physiology of renal recovery, a number of biomarkers have the potential to predict renal recovery. Neutrophil gelatinase-associated lipocalin (NGAL) has been extensively studied in the field of AKI. In the late phase of AKI, NGAL is believed to play a role as a growth and differentiation factor for restoring tubular epithelial function with the assistance of siderophore-iron complexes. However, most of the previous studies have tested plasma/urine NGAL as a marker for the early diagnosis of AKI, and only a few studies have examined plasma/ urine NGAL as a prognostic marker of clinical outcome.

We examined the plasma of patients on the first day on which they experienced severe AKI (defined as RIFLE-F) [32]. Elevated pNGAL levels were associated with renal nonrecovery, although the absolute predictive value of pNGAL alone was only fair (area under the receiver operating characteristics curve $=0.74$ ). These data are most notable for two reasons. First, because AKI can cause $\mathrm{CKD}$ and end-stage renal disease, decisions regarding long-term care (e.g. use of dialysis, vascular access, and follow-up) are often made using a piecemeal approach. If objective metrics coupled with clinical assessment can improve the prognostic accuracy, a better-informed decision can be made for survivors of AKI. Second, the association between pNGAL and renal nonrecovery suggests that renal injury is ongoing and, even if a patient is undergoing dialysis, therapies directed at mitigating ongoing injury may have a role in AKI treatment [32].

We also conducted the Biological Markers of Recovery for the Kidney (BioMarK) study as an ancillary to the ATN study [33]. Urine samples were collected on days 1, 7, and 14 from 76 patients who developed AKI and required renal support. We explored whether levels of 
uNGAL, uHGF, uCystatin C, uIL-18, uNGAL/MMP-9, and urine creatinine could predict subsequent renal recovery. Patients who recovered had a higher uCystatin C level on day 1 and lower uHGF levels on days 7 and 14 . For predicting recovery, decreasing uNGAL and uHGF levels in the first 14 days were associated with greater odds of renal recovery.

Using the BioMarK cohort, we also examined 11 plasma inflammatory and apoptosis markers on renal recovery and mortality in 817 critically ill subjects receiving RRT [34]. The study found that increased concentrations of plasma IL-8, IL-18, and TNFR-I were independently associated with a slower renal recovery (adjusted hazard ratio range for all markers $=0.70-0.87)$. Higher concentrations of IL-6, IL-8, IL-10, IL-18, MIF, TNFR-I, and DR-5 were associated with mortality. In an analysis of multiple markers simultaneously, increased IL-8 and TNFR-I were associated with a slower recovery, and increased IL-8, MIF, and TNFR-I were associated with mortality.

Finally, markers of cell cycle arrest were recently shown to predict human AKI $[35,36]$. IGF-binding protein (IGFBP)-7 and tissue inhibitor of metalloproteinase (TIMP)-2 are both linked to cell cycle arrest during the very early phases of cell injury. In response to cell stress, IGFBP7 and TIMP2 are expressed in the tubular cells. IGFBP7 directly increases the expression of p53 and p21 and TIMP2 stimulates p27 expression. These effects are conducted in an autocrine and paracrine manner and the $\mathrm{p}$ proteins, in turn, block the effect of cyclin-dependent protein kinase complexes (CyclD-CDK4 and CyclECDK2) on cell cycle promotion, thereby resulting in transient cell cycle arrest. It is likely that this effect is protective when temporary and helps avoid injured cells from dividing - which can result in apoptosis of both daughter cells. However, prolonged cell cycle arrest may lead to maladaptive repair and fibrosis. Supporting this, Kashani et al. [35], using a large cohort of critically ill patients at risk for AKI, found that a urinary [TIMP-2] · [IGFBP7] value above 2.0 was associated with almost a 4 -fold increased risk of major adverse kidney events at 30 days. Major adverse kidney events were defined as the composite of death, use of renal replacement therapy, or persistence of renal dysfunction (defined by serum creatinine levels $\geq 200 \%$ of the reference) at hospital discharge truncated at 30 days [35].

\section{Conclusions}

Increasing evidence demonstrates that AKI may result in important long-term adverse outcomes. Therefore, methods to identify which patients are likely to develop this complication are urgently needed, as are treatments to mitigate this risk. Biomarkers may also be useful in predicting renal recovery and may therefore play an important role in disease management.

\section{References}

1 VA/NIH Acute Renal Failure Trial Network, Palevsky PM, et al: Intensity of renal support in critically ill patients with acute kidney injury. N Engl J Med 2008;359:7-20.

2 RENAL Replacement Therapy Study Investigators, Bellomo R, et al: Intensity of continuous renal-replacement therapy in critically ill patients. N Engl J Med 2009;361:1627-1638.

3 Bellomo R, et al; Acute Dialysis Quality Initiative workgroup: Acute renal failure - definition, outcome measures, animal models, fluid therapy and information technology needs: the Second International Consensus Conference of the Acute Dialysis Quality Initiative (ADQI) Group. Crit Care 2004;8: R204-R212.

4 Shibe S, Cantley LG: Epithelial-mesenchymal-epithelial cycling in kidney repair. Curr Opin Nephrol Hypertens 2008;17:379-385.

5 Liu KD, Brakeman PR: Renal repair and recovery. Crit Care Med 2008;36:S187-S192.
6 Singbartl K, Kellum JA: AKI in the ICU: definition, epidemiology, risk stratification, and outcomes. Kidney Int 2012;81:819-825.

7 Sirin Y, Susztak K: Notch in the kidney: development and disease. J Pathol 2012;226:394403.

8 McCright B: Notch signaling in kidney development. Curr Opin Nephrol Hypertens 2003; 12:5-10.

9 Kobayashi T, et al: Expression and function of the Delta-1/Notch-2/Hes-1 pathway during experimental acute kidney injury. Kidney Int 2008;73:1240-1250.

10 Gupta S, et al: Effect of Notch activation on the regenerative response to acute renal failure. Am J Physiol Renal Physiol 2010;298: F209-F215.

11 Bielesz B, et al: Epithelial Notch signaling regulates interstitial fibrosis development in the kidneys of mice and humans. J Clin Invest 2010;120:4040-4054.
12 Yang L, et al: Epithelial cell cycle arrest in G2/M mediates kidney fibrosis after injury. Nat Med 2010;16:535-543.

13 Cianciolo C, et al: Histone deacetylase inhibitor enhances recovery after AKI. J Am Soc Nephrol 2013;24:943-953.

14 Zhou L, et al: Activation of $\mathrm{p} 53$ promotes renal injury in acute aristolochic acid nephropathy. J Am Soc Nephrol 2010;21:31-41.

15 Bhandari S, Turney JH: Survivors of acute renal failure who do not recover renal function. QJM 1996;89:415-421.

16 Uchino S, et al, Beginning and Ending Supportive Therapy for the Kidney (BEST Kidney) Investigators: Acute renal failure in critically ill patients: a multinational, multicenter study. JAMA 2005;294:813-818.

17 Bell $\mathrm{M}$, et al: Continuous renal replacement therapy is associated with less chronic renal failure than intermittent haemodialysis after acute renal failure. Intensive Care Med 2007; 33:773-780. 
18 Uchino S, et al: Continuous renal replacement therapy: a worldwide practice survey - the beginning and ending supportive therapy for the kidney (BEST kidney) investigators. Intensive Care Med 2007;33:1563-1570.

19 Lo LJ, et al: Dialysis-requiring acute renal failure increases the risk of progressive chronic kidney disease. Kidney Int 2009;76:893-899.

20 Choi AI, et al: Long-term clinical consequences of acute kidney injury in the HIVinfected. Kidney Int 2010;78:478-485.

21 Newsome BB, et al: Long-term risk of mortality and end-stage renal disease among the elderly after small increases in serum creatinine level during hospitalization for acute myocardial infarction. Arch Intern Med 2008; 168 609-616.

22 James MT, et al: Acute kidney injury following coronary angiography is associated with a long-term decline in kidney function. Kidney Int 2010;78:803-809.

23 James MT, et al; Alberta Kidney Disease Network: Glomerular filtration rate, proteinuria, and the incidence and consequences of acute kidney injury: a cohort study. Lancet 2010 376:2096-2103.
24 Wald R, et al; University of Toronto Acute Kidney Injury Research Group: Chronic dialysis and death among survivors of acute kidney injury requiring dialysis. JAMA 2009;302: 1179-1185.

25 Lafrance JP, et al: Incidence and outcomes of acute kidney injury in a referred chronic kidney disease cohort. Nephrol Dial Transplant 2010;25:2203-2209.

26 Ishani A, et al: Acute kidney injury increases risk of ESRD among elderly. J Am Soc Nephrol 2009;20:223-228.

27 Ishani A, et al: The magnitude of acute serum creatinine increase after cardiac surgery and the risk of chronic kidney disease, progression of kidney disease, and death. Arch Intern Med 2011;171:226-233.

28 Amdur RL, et al: Outcomes following diagnosis of acute renal failure in US veterans: focus on acute tubular necrosis. Kidney Int 2009;76: 1089-1097.

29 Uchino S, et al: External validation of severity scoring systems for acute renal failure using a multinational database. Crit Care Med 2005; 33:1961-1967.

30 Macedo E, et al: Oliguria is an early predictor of higher mortality in critically ill patients. Kidney Int 2011;80:760-767.

31 Uchino S, et al: Discontinuation of continuous renal replacement therapy: a post hoc analysis of a prospective multicenter observational study. Crit Care Med 2009;37:25762582.
32 Srisawat N, et al; Genetic and Inflammatory Markers of Sepsis (GenIMS) Study Investigators: Plasma neutrophil gelatinase-associated lipocalin predicts recovery from acute kidney injury following community-acquired pneumonia. Kidney Int 2011;80:545-552.

33 Srisawat N, et al: Urinary biomarkers and renal recovery in critically ill patients with renal support. Clin J Am Soc Nephrol 2011;6:18151823.

34 Murugan R, et al: Plasma inflammatory and apoptosis markers are associated with dialysis dependence and death among critically ill patients receiving renal replacement therapy. Nephrol Dial Transplant 2014, DOI: 10.1093/ ndt/gfu051.

35 Kashani K, et al: Discovery and validation of cell cycle arrest biomarkers in human acute kidney injury. Crit Care 2013;17:R25.

36 Bihorac A, et al: Validation of cell-cycle arrest biomarkers for acute kidney injury using clinical adjudication. Am J Respir Crit Care Med 2014;189:932-939.

37 Srisawat N, et al: Recovery from acute kidney injury: determinants and predictors. Contrib Nephrol 2010;165:284-291. 